\title{
Vitamin D: The Need of the Hour
}

\author{
Praveen Sharma ${ }^{1} \cdot$ Prasenjit Mitra $^{1}$
}

Published online: 20 May 2016

(C) Association of Clinical Biochemists of India 2016

Vitamin D has been in limelight for the last decade because of its connection with several health disorders. With time, there is remarkably increasing evidence of vitamin D status being associated with the risk of several diseases including but not limited to diabetes mellitus, osteoarthritis, metabolic syndrome, cancer, immunological diseases, respiratory diseases, cardiovascular diseases etc. The concern has further increased as most of the studies done globally have revealed the prevalence of vitamin D deficiency in a larger fraction of population [1]. In India, most observational studies have reported similar trends. It is quite surprising that vitamin D which is also known as 'sunshine vitamin' is deficient in a large group of people irrespective of their exposure to ample amounts of sunlight throughout the year [2].

Vitamin D deficiency in such alarming numbers have increased the urge in clinicians to start vitamin D supplementation. This has led to an increase in the number of vitamin $\mathrm{D}$ preparations in market and also newer formulations and combinations are being released by various manufacturers. In some places, vitamin D is being supplemented even without knowing its status in an individual. This is because facility for vitamin D estimation is limited mostly to tertiary health care providers or private sectors and only few with high income can get themselves checked as a part of routine health check-up. For others, it's cheaper to pop a vitamin D pill and get supplemented instead of getting diagnosed. But the downside of this practise includes exposure to various toxic effects. A global debate

Praveen Sharma

praveensharma55@gmail.com

1 Department of Biochemistry, All India Institute of Medical Sciences, Jodhpur, India has already started on the utility of screening for vitamin D deficiency versus its supplementation blindly [3]. The requirement of vitamin $\mathrm{D}$ in an individual and its healthy level is also a subject of debate. As per Institute of Medicine (IOM), a serum vitamin D value of $20 \mathrm{ng} / \mathrm{ml}$ is considered optimal in human adults whereas according to the U.S. Endocrine Society $30 \mathrm{ng} / \mathrm{ml}$ is optimal $[4,5]$. Due to this variation the recommended daily intakes of this nutrient also vary.

In Indian studies, where a serum vitamin D value of $<30 \mathrm{ng} / \mathrm{ml}$ was considered as diagnostic criteria for deficiency, almost entire study population were found to be vitamin $\mathrm{D}$ deficient irrespective of diverse age groups, gender and other demographic factors [6]. If a nutrient is deficient in a large number of otherwise healthy population, naturally question arises on the validity of diagnostic test and more importantly on the reliability of reference range being used for interpretation of results. Most of the observational studies done in India have considered reference ranges of vitamin $\mathrm{D}$ as per international recommendations or as per mentioned by the manufacturers of commercial kits. Very few researchers have actually tried to determine the reference range of vitamin D and standardise the cut-off values in Indian population. The geographical nature and the highly diverse Indian population with respect to culture, ethnicity and socio-economic status is bound to have an impact on the vitamin D status throughout the country which can only become evident if proper measures are taken to establish its reference range in this population. Till then, it becomes difficult to accept logically the prevalence of vitamin D deficiency in such large numbers and equally difficult to reject the same scientifically. All these facts strongly suggests the urgent need to review the reference range of vitamin D in Indian population and standardise the cut-off values for its deficiency. 
It is a good news that Indian Council of Medical Research (ICMR) has taken initiative to explore vitamin D status in a large group of Indian population. It will definitely provide directions to set up new guidelines for requirement and optimal levels of vitamin D in this population. Till then, the best solution for this problem is probably setting up of own reference range by the laboratories involved in the estimation of this vital nutrient.

\section{References}

1. Holick MF. Vitamin D deficiency. N Engl J Med. 2007;357: 266-81.
2. Trilok Kumar G, Chugh R, Poor Eggersdorfer M. Vitamin D Status in healthy populations in India: a review of current evidence. Int J Vitam Nutr Res. 2015;85(3-4):185-201.

3. LeBlanc ES, Zakher B, Daeges M, Pappas M, Chou R. Screening for vitamin D deficiency: a systematic review for the U.S. Preventive Services Task Force. Ann Intern Med. 2015;162:109-22.

4. Balvers MG, Brouwer-Brolsma EM, Endenburg S, de Groot LC, Kok FJ, Gunnewiek JK. Recommended intakes of vitamin D to optimise health, associated circulating 25-hydroxyvitamin D concentrations, and dosing regimens to treat deficiency: Workshop report and overview of current literature. J Nutr Sci. 2015;4:e23.

5. Ross A, Manson J, Abrams S, Aloia J, Brannon P, Clinton S, et al. The 2011 report on dietary reference intakes for calcium and vitamin D from the Institute of Medicine: what clinicians need to know. J Clin Endocrinol Metab. 2011;96(1):53-8.

6. Ritu G, Gupta A. Vitamin D deficiency in India: prevalence, causalities and interventions. Nutrients. 2014;6(2):729-75. 